\title{
A randomized clinical trial of topical treatments for mild and severe udder cleft dermatitis in Dutch dairy cows
}

\author{
T. van Werven, ${ }^{*} \dagger^{1}$ J. Wilmink, $\ddagger$ S. Sietsma, $\dagger$ J. van den Broek, ${ }^{*}$ and M. Nielen* \\ *Department of Farm Animal Health, Faculty of Veterinary Medicine, Utrecht University, 3584 CL, Utrecht, the Netherlands \\ †University Farm Animal Practice, 3481 LZ, Harmelen, the Netherlands \\ fWoumarec, 6705 CT, Wageningen, the Netherlands
}

\begin{abstract}
Udder cleft dermatitis (UCD) is a skin lesion in dairy cows affecting the anterior parts of the udder, with the lesions often needing a long time to heal. The lesions can be characterized as mild or severe. The etiology of UCD is not fully understood and studies on the effectiveness of topical treatments have not been published. The objective of this study, therefore, was to conduct a randomized clinical trial to investigate the effectiveness of 2 different topical treatments, one for mild and one for severe UCD lesions, compared with untreated control groups. The treatment and control groups were randomized within herd for mild and severe UCD. The treatments were applied for a maximum period of 12 wk on 8 Dutch dairy farms. Mild UCD lesions were treated once a d 3 times a week on fixed days with a non-sting barrier film. Severe UCD lesions were first stratified into class A (lesion length $<5 \mathrm{~cm}$ ) or class $\mathrm{B}$ (lesion length $\geq 5 \mathrm{~cm}$ ) and then randomly allocated to treatment or control groups within herd. Both severe lesion classes were treated once per day every day with an enzyme alginogel. Every week, the lesions of affected animals were inspected and photographed by the investigator. These photographs were reviewed weekly by an external wound expert who classified the lesions as mild, severe class A, severe class B, or healed. Based on this classification, the investigator judged weekly whether the lesions had improved compared with their classification of the previous week. For mild UCD lesions, improvement was defined as occurring when lesions were healed. For severe UCD lesions, improvement was defined as a transition from class B to class A, transition from any severe UCD lesion (class A or B) to a mild UCD lesion, or when the lesion was defined as healed. Data were analyzed using a discrete time survival analysis with time to first improvement as dependent variable. In total, data from 214 animals
\end{abstract}

Received September 1, 2017.

Accepted May 24, 2018.

${ }^{1}$ Corresponding author: T.vanWerven@uu.nl were analyzed to estimate the effectiveness of treatment. Results showed that treatment of mild UCD lesions had no influence on improvement compared with untreated lesions. Treated severe lesions, however, showed 3.4 times more improvement compared with the untreated controls. Improvement varied between herds, and cows with a parity of 5 or higher showed significantly less improvement than first parity animals. Early identification of severe UCD lesions followed by prompt treatment with an enzyme alginogel supports the healing process.

Key words: udder cleft dermatitis, dairy cow, topical treatment, randomized clinical trial

\section{INTRODUCTION}

Skin lesions in dairy cows located between the front quarters of the udder or at the anterior junction between the udder and abdomen are known as udder cleft dermatitis (UCD). Udder cleft dermatitis lesions have been reported in the United Kingdom (Beattie and Taylor, 2000), United States (Warnick et al., 2002), Sweden (Persson Waller et al., 2014; Ekman et al., 2018), and the Netherlands (Bouma et al., 2016). The herd prevalence ranges between 0 and $43 \%$ and the severity of UCD lesions varies between herds. Udder cleft dermatitis lesions heal poorly (Bouma et al., 2016) and have been shown to increase the risk of embolic pneumonia (Millar et al., 2017), affecting both animal health and animal welfare.

The etiology of UCD is not fully understood. Some studies suggest the involvement of infectious agents, such as sarcoptic mange (Warnick et al., 2002) or Treponema (Stamm et al., 2009; Evans et al., 2010). However, a Dutch study could not identify any involvement of Treponema, Escherichia coli, Staphylococcus aureus, yeast, or fungi (van Engelen et al., 2014). The Dutch study did detect Bacteroides pyogenes and Trueperella pyogenes significantly more often in severe UCD lesions compared with mild UCD lesions. The presence of these opportunistic anaerobic bacteria seems more indicative of secondary bacterial involvement than of 
a primary bacterial infection. Mechanical factors that could irritate the skin such as certain udder conformation traits (Olde Riekerink et al., 2014; Ekman et al., 2018), or housing- related factors such as shorter cubicles or matrasses as cubicle base (Ekman et al., 2018) have been identified as risk factors. Other risk factors that have been identified are more DIM (Bouma et al., 2016; Ekman et al., 2018) and breed and parity (Persson Waller et al., 2014; Bouma et al., 2016; Ekman et al., 2018). As yet a causal mechanism has not been identified, but a multi-factorial etiology is most likely.

To date, no proven effective treatment studies on UCD have been reported. The lack of an effective treatment often results in the field in ineffective treatments or untreated UCD lesions. When UCD lesions are not treated, the exudate desiccates and turns into crusts, which is known to be disadvantageous because wounds need moisture to heal (Winter, 1962). Udder cleft dermatitis lesions or intertrigo show many similarities in morphology and healing to human pressure ulcers. Mild UCD lesions look similar to stage 1 pressure ulcers, whereas severe UCD lesions are comparable to stage 2, 3, and 4 lesions (Black et al., 2007). Both pressure ulcers and UCD lesions show delayed healing, which is often caused by cellular and molecular imbalances in the wound bed (Eming et al., 2007). Particularly for hard-to-heal wounds it is important to adapt the treatment to the requirements of the wound and the phase of healing (Wilmink, 2017). In human medicine, some topical treatments have given good results for the different types of pressure ulcers, but these treatments have not been tested for UCD lesions in dairy cows.

Therefore, the aim of our study was to perform a randomized clinical trial to assess the clinical effectiveness of 2 different topical treatments, one for mild and one for severe UCD lesions, compared with untreated control groups. None of treatments contained antibiotics and the choice of treatments was based on evidencebased research in human medicine (Cameron et al., 2005; Beele et al., 2012; Chan and Siu, 2016). The topical treatment for mild UCD lesions consisted of a nonalcoholic film layer and focused on the protection of the lesion from moisture and dirt. Topical treatment of the severe lesions consisted of an enzyme alginogel and was directed toward stimulation of the inflammatory response and wound healing, and toward restoration of the bacterial balance.

\section{MATERIALS AND METHODS}

A randomized clinical trial was carried out on 8 Dutch dairy herds and for logistical reasons conducted in 2 rounds, each consisting of 4 participating dairy herds.
A treatment period of maximum 12 wk per round was carried out based on the results of a longitudinal study (Bouma et al., 2016) that showed a median observed duration of $8.8 \mathrm{wk}$ for mild UCD lesions and $16.2 \mathrm{wk}$ for severe UCD lesions. Round 1 took place from May 12, 2014, until August 22, 2014, and round 2 from September 30, 2014, until January 9, 2015.

\section{Herd Selection}

Eight commercial dairy herds, 7 within one practice centrally located in the Netherlands (University Large Animal Practice, Harmelen, the Netherlands), and one herd in the neighboring practice (Amstel, Vecht en Venen, Vinkeveen, the Netherlands), were selected based on a total UCD prevalence of at least $6 \%$, the likelihood of compliance with the study protocol, and enrolled in a DHI program every 4 or 6 wk. Herds with automatic milking systems were excluded because daily treatment of the affected animals by the farmer was considered to be too labor intensive in those herds.

This intervention study was approved by the Ethical Committee of Utrecht University, the Netherlands, and was not deemed to be an animal experiment under Dutch Law (email March 3, 2014).

\section{Cow Selection}

All dairy cows (dry and lactating) in the herd were eligible for enrollment in the trial. Cows were selected during the recruitment visit (T0), when all animals were inspected for signs indicative of UCD. Farms were visited just after morning milking, and all dry and lactating cows were fixed into headlocks and visually inspected one by one. The investigator used a lamp and hand mirror in one hand and spread the front quarters of the udder with the other hand for proper inspection of the skin between the 2 front quarters and the skin of the anterior junction between the udder and the abdominal wall. If signs indicative of UCD were present, a ventral photograph was taken via a mirror as described elsewhere (Bouma et al., 2016). If no signs indicative of UCD were observed, the udder was not photographed and the cow was not eligible to participate in the study.

\section{UCD Classification}

Skin integrity distinguished mild UCD lesions from severe UCD lesions, as this appeared to be the most distinctive characteristic for categorizing UCD (Persson Waller et al., 2014; Bouma et al., 2016). A UCD lesion was defined as mild when symptoms such as erythema, transudate, sebum, crusts, or scar tissue were present 
combined with intact skin integrity or when there was a lesion with only a small area of granulation tissue of $<2 \mathrm{~cm}^{2}$.

A UCD lesion was defined as severe when the skin barrier was broken and an open wound was present with either necrosis, blood clots, or granulation tissue and exudate. The severe UCD lesions were additionally subdivided into 2 classes depending on the length of the lesion (class A lesion $<5 \mathrm{~cm}$, class B lesion $\geq 5 \mathrm{~cm}$; Appendix Figure A1).

\section{Study Protocol}

Treatment Allocation. After the photographs from the recruitment visit were scored by the external wound expert, mild UCD cases were randomly allocated to a treatment and control group within herd. Within herd, affected cows with mild UCD lesions were sorted by cow number. The first cow on the list was allocated to the treatment group and thereafter cows were allocated one by one alternately to either the control or treatment groups. Within herd the severe UCD lesions were first divided into class A and B based on the length of the lesion. Within class A and class B, animals were randomly allocated to a treatment and control group within the herd as described above for mild UCD lesions.

Treatment Procedures. After allocation of the animals to the treatment and control groups, herds were visited again (at wk 0) within 2 wk after the recruitment visit. Cows to be included in the treatment group were identified by colored leg bands. Animals in the mild UCD treatment group got a yellow leg band and the animals in the severe UCD treatment group were identified with a blue band. Cows in both treatment groups were not treated during the dry period as the treatments had to be applied in the milking parlor, but treatment started or started again immediately after calving. Animals in the control groups were not identified for the farmer because these cows did not receive any treatment.

First treatments were applied by the investigator at wk 0. After identification of the animal, the affected skin was cleaned carefully with a wet paper towel, available in the milking parlor for udder preparation, and the crust and necrotic tissue were removed by the investigator. Finally, the skin and wound tissue were dried thoroughly with a paper towel and the treatments were applied by the investigator, in line with the treatment allocation. Farmers were shown how to clean the lesions before each treatment with a dry paper towel and the application of the 2 different topical treatments was demonstrated to them. All mild and severe UCD le- sions in the treatment groups were treated until healed or for a maximum of $12 \mathrm{wk}$, starting at wk 0 until wk 12. Mild and severe lesions in the control group were left untreated.

Treatments. The trial consisted of 2 different topical treatments, one for the mild and one for the severe UCD cases. All treatments, except for the first one at wk 0, were applied by the farmers in the milking parlor. Mild UCD cases were treated with an alcohol-free barrier film (Cavilon, 3M, Maplewood, MN), which provided a nonalcoholic film layer over the damaged skin. A small $1 \times 1 \mathrm{~cm}$ tissue saturated with the film was wiped over the lesion. The film layer was applied once per day after milking on Monday, Wednesday, and Friday or on Tuesday, Thursday, and Saturday. The coating or film protects the skin from contamination and irritation by dirt in the environment (feces, urine, bedding, and so on) and reduces friction (Cameron et al., 2005; Holroyd and Graham, 2014; Chan and Siu, 2016).

Severe UCD lesions were treated with an enzyme alginogel (BoTop, Flen Health, London, UK) once every day after milking. A small amount of the alginogel, approximately 5 to $10 \mathrm{~g}$ depending on the lesion size, was divided over 2 fingertips and spread gently over the severe lesions, using a clean glove for each treatment. The enzyme alginogel consists of a hydrated alginate, polyethylene glycol, and an antimicrobial enzyme system, and simultaneously exhibits autolytic, absorbent, and antimicrobial activities (Hämmerle and Strohal, 2015).

UCD Observation Protocol for Follow-Up. After the initial treatment by the investigators at wk 0 , thereafter farms were visited once every week by the investigator from wk 1 to 12 . Farms were visited just after morning milking, cows were fixed into headlocks, and all affected animals were individually inspected and photographed. Because cows were treated for the first time at wk 0 , improvement of the lesions related to the treatment could be measured from wk 1 until wk 12. Every week, the photographs from the affected animals were sent electronically to the external wound specialist who was blinded to the group allocation. The photographs were evaluated according to the classification system into mild or severe lesions. The length of the lesion was measured by the wound expert with the help of a ruler that was photographed parallel to the lesion by the investigator and was used to stratify severe lesion into class A $(<5 \mathrm{~cm})$ or class B $(\geq 5 \mathrm{~cm})$. A file with the classification results was sent back to the investigator within $1 \mathrm{wk}$.

Every week the investigator compared the classification results from the external wound expert with the 
classification results from the previous week and decided whether the lesions had improved, deteriorated, or shown no change. For a mild UCD case, improvement was defined as having taken place when the lesion was characterized as healed. First improvement of severe UCD cases was defined as transition from class B to class A or transition from severe UCD (class A or B) to mild UCD or to complete healing. All animals with lesions that were classified as healed were no longer followed up.

If the lesions from animals in the treated groups had moved from severe to mild or vice versa, during the next farm visit the following week the relevant animals were moved to the relevant treatment group, in line with the newest characterization and leg bands from the animals were changed accordingly. If the lesions from animals in the control groups moved from severe to mild or vice versa, they stayed in the untreated control group.

Assessment of Effectiveness. The primary outcome of the study was defined as time to first improvement of the mild or severe UCD cases as classified at the recruitment visit T0. First improvement was defined as given by the definition above.

Analysis was based within the original mild or severe classification during the recruitment visit. The effectiveness of the 2 topical treatments, one for mild and one for severe UCD cases, was compared with their respective mild and severe control groups (no treatment).

\section{Data Recording}

The duration of the treatment was a maximum of $12 \mathrm{wk}$, less if the lesions were defined as healed. Data on lesion classification executed by the external wound specialist were entered into a spreadsheet using MS Excel (Microsoft Office, Redmond, WA) per herd, whereas electronic copies of the photographs were stored on a computer in herd-specific folders. The photos were mirrored using PhotoFiltre software (version 7.1.2, Da Cruz, Houilles, France) and stored as jpg files. Every week these jpg files were sent to the external wound specialist to enable her to categorize the lesions into mild, severe A, severe B, or healed. The categories were sent back to the investigator, entered into Excel, and used to judge whether lesions had improved, deteriorated, or remained unchanged. Based on the specialist categories, a weekly UCD status was recorded for all participating cows. Cow specific data such as parity and DIM were collected from the milk recordings.

\section{Power Calculation}

The power calculation was based on the number of mild UCD cases to be included. Estimations were based on the results of a longitudinal study on UCD (Bouma et al., 2016), which estimated that the probability of moving from any UCD case to a healthy animal between 2 consecutive visits was $5.5 \%$ per week. Assuming that the application of mild UCD treatment increases the probability of moving from a UCD case to a healed case to $25 \%$, the study would require 56 animals per group (power $85 \%$ and CI 95\%, 2-sided test).

\section{Statistical Analyses}

First, descriptive analyses were done on the number of enrolled animals within the 8 participating herds and divided over the mild and severe UCD cases, including baseline values for potential confounders. Proportion of first improvement are reported for mild and severe lesions per treatment group, and proportions per severity group were tested by chi-squared test.

Before statistical analysis, descriptive analyses were studied and showed that 8 animals with mild UCD lesions at T0 showed first improvement during the follow-up period only after switch toward severe lesions. These 8 mild lesions were censored from the time point they became severe onward, precluding further statistical analysis (Figure 1). Animals that were culled or removed from the trial because of heavily kicking were also censored from that time point onward.

The time to first improvement was analyzed with a discrete time survival analysis because time steps consisted of whole weeks. This survival analysis was technically modeled in a logistic regression model with time step as a discrete additional parameter (Burnham and Anderson, 2002; Dohoo et al., 2009). The full model statement for the log-odds of the hazard probability of first improvement was as follows:

$$
\begin{gathered}
\operatorname{logit} \mathrm{h}(\mathrm{t})=\beta_{0}+\beta_{1} \times \text { StatusT0 } \\
+\beta_{2} \times \operatorname{TreatM}_{(\text {StatusT0) }}+\beta_{3} \times \operatorname{TreatS}_{(\text {StatusT0 })} \\
+\beta_{4-10} \times \text { Farm2-8 }+\beta_{11-14} \times \text { Par2 }-5+\beta_{15} \\
\times \text { Round }+\beta_{16} \times \operatorname{Dry}(\text { wk })+\beta_{17} \times \text { DIM } \\
+\left[\beta_{18} \times \text { wk } 2 \ldots \beta_{28} \times \text { wk } 12\right],
\end{gathered}
$$

where logit $\mathrm{h}(\mathrm{t})=$ conditional probability that improvement occurs at week $t$ given that it has not occurred before; $\beta_{0}=$ intercept; $\beta_{1}$ to $\beta_{28}=$ parameter estimate; StatusT0 $=$ lesion status mild or severe at T0 (reference $=$ mild); $\operatorname{TreatM}_{(\mathrm{StatusT0})}$ and Treat $_{(\mathrm{StatusT0})}=$ treatment yes/no within status at T0: mild Cavilon and severe BoTop; Farm 1-8 as fixed effect (reference $=1$ ); Par $=$ parity $1,2,3,4$, and $\geq 5$ (reference $=1)$; Round $=1,2($ reference $=1) ; \operatorname{Dry}($ wk $)=$ time varying vari- 


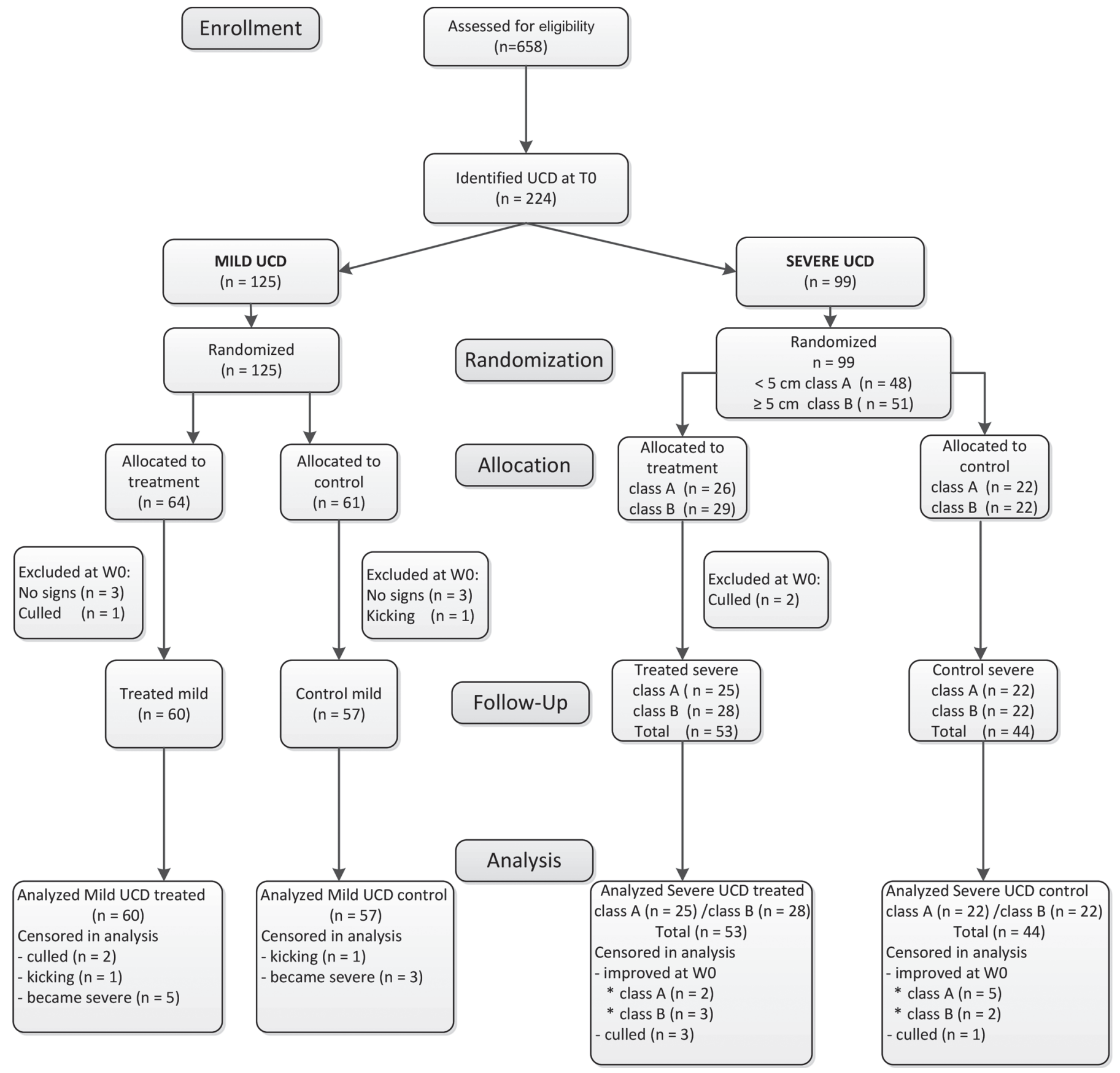

Figure 1. Flow diagram of the recruited animals in the randomized clinical trial of topical treatments for mild and severe udder cleft dermatitis (UCD) lesions from 8 herds in the Netherlands, with a total of 214 affected animals analyzed (117 mild UCD lesions and 97 severe UCD lesions).

able dry period yes/no per week; DIM = days in milk at $\mathrm{T} 0$; wk $=$ week in follow up $1-12($ reference $=1)$.

Week needs to be in the model to estimate a baseline odds function. Dry period was in the model as a time-varying covariate (Silveira Chalita et al., 2006; Dohoo et al., 2009). Because the mild UCD lesions were treated with Cavilon and the severe lesions with Bo-
Top, treatment was modeled as a nested effect within status at T0. This means that for each status at T0 (mild or severe), a separate treatment effect was estimated; untreated and treated mild lesions were compared with each other, as well as untreated and treated severe lesions. Collinearity was checked at the start of the model building process and deviance residual was 
Table 1. Characteristics of the 8 Dutch dairy herds participating in the randomized clinical trial on udder cleft dermatitis (UCD) and the number of included animals at the recruitment visit (T0) on each farm on which statistical analysis was performed $(\mathrm{n}=214)$

\begin{tabular}{|c|c|c|c|c|c|}
\hline Herd $^{1}$ & $\begin{array}{l}\text { Herd size } \\
\quad(\mathrm{T} 0)\end{array}$ & $\begin{array}{l}\text { Number of recruited } \\
\text { mild UCD cases }\end{array}$ & $\begin{array}{l}\text { Number of recruited } \\
\text { severe UCD cases }\end{array}$ & $\begin{array}{l}\text { 305-d milk } \\
\text { yield }(\mathrm{kg})\end{array}$ & $\begin{array}{l}\text { Bedding material } \\
\text { in cubicles }\end{array}$ \\
\hline 1 & 65 & 18 & 13 & 8,709 & Sawdust and lime \\
\hline 4 & 83 & 15 & 6 & 9,720 & Straw, water, and lime \\
\hline 5 & 120 & 13 & 15 & 8,221 & Straw, sawdust, and lime \\
\hline 6 & 68 & 10 & 21 & 10,540 & Sawdust \\
\hline
\end{tabular}

${ }^{1}$ Herds $1,6,7$, and 8 participated in period 1 and herds $2,3,4$, and 5 participated in period 2 of the trial.

used for model checking. Model reduction was carried out using a backward method with Akaike's information criterion. The $95 \%$ profile (log) likelihood intervals were calculated for the important effects in the model. The discrete time survival analysis was applied in $\mathrm{R}$ version 3.3.0 (R Core Team, 2016, R Foundation for Statistical Computing, Vienna, Austria).

\section{RESULTS}

\section{Descriptive Data}

From the 658 cows present in the 8 herds that were assessed for eligibility, a total of 224 cows were recruited to the trial during the first recruitment visit at T0 (Figure 1). Of these 224 animals, 125 cows (56\%) showed signs of mild UCD lesions and 99 animals (44\%) showed signs of severe UCD. Within herd, the 125 animals with mild UCD signs were randomly allocated to the treatment group $(\mathrm{n}=64)$ or the control group $(\mathrm{n}=61)$. Of the 99 severe UCD cases, 48 lesions were classified as class $\mathrm{A}(<5 \mathrm{~cm})$ and 51 lesions as class B $(\geq 5 \mathrm{~cm})$. Of the 48 animals with severe class A UCD lesions, 26 animals were randomly allocated to the treatment group and 22 animals to the control group. The 51 animals with class B severe UCD lesions were randomly allocated to the treatment group $(\mathrm{n}=$ $29)$ and the control group $(\mathrm{n}=22$; Figure 1$)$.

Within 2 wk after the recruitment visit, farms were visited again at wk 0 to start the treatment. During inspection of affected animals, a total of 10 animals were excluded from the trial. In 6 animals with mild UCD lesions the clinical symptoms of UCD had disappeared ( 3 allocated to the treatment group and 3 to the control group), 1 animal with mild UCD lesions was heavily kicking and therefore removed from the trial, and 3 animals were culled in the meantime ( 1 with mild and 2 with severe UCD lesions; Figure 1).

This finally resulted in 214 animals divided over 117 mild UCD cases (60 treatment and 57 control) and
97 severe UCD cases from which 53 in the treatment group (25 class A and 28 class B lesions) and 44 in the control group (22 class A and 22 class B lesions). The characteristics of each of the farms and the number of cows recruited on which analysis was performed are shown in Table 1. Percentage of enrolled animals with UCD lesions per herd showed a range between 10\% (herd 2) to $48 \%$ (herd 1 and herd 8).

Baseline values that may influence UCD lesion healing, such as parity and DIM within the different UCD groups (mild, severe class A, and severe class B), are shown in Table 2. The median DIM of cows with severe UCD lesions was more than $100 \mathrm{~d}$ shorter than the median DIM of cows with mild UCD lesions.

\section{First Improvement of Lesions}

A first improvement of the mild UCD lesions was observed in 21 out of $60(35 \%)$ treated mild cases, compared with 20 out of $57(35 \%)$ control cases within the 12 -wk follow-up period. Within the animals with severe UCD lesions, 13 animals already showed improvement at wk 0 before the start of the treatment period ( 6 in the treatment group and 7 in the control group) as shown in Figure 1. Table 3 shows first improvement related to therapy from wk 1 until wk 12 . First improvement in severe UCD lesions was observed in 28 out of $47(60 \%)$ treated cases compared with 12 out of $37(32 \%)$ severe lesions in the control group. The treated severe UCD lesions in class A showed significantly more first improvement than the treated severe UCD lesions in class B, 78 versus $47 \%$, respectively $(P<0.05)$. Subsequent healing was only observed in 7 severe class A lesions (6 treated cases) and in 3 severe class B cases (2 treated cases).

Median time to first improvement for mild UCD lesions was observed at wk 4 (i.e., after 4 wk of treatment) for both the treatment and control groups. The severe class A lesions showed a median time to first improvement at wk 3 in the treatment group and at wk 
4 in the control group. Median time to first improvement for the severe class B lesions was observed at wk 1 in the treatment group and between wk 1 and 2 in the control group.

\section{Model Results}

Eight animals in the treatment group with mild UCD lesions at the recruitment visit changed during the trial into severe UCD lesions and were therefore censored at that time point in the discrete time survival analysis, because of treatment switch. According to the Akaike's information criterion, the following effects were important in the model: farm, parity, dry period, and treatment within status at T0. The backward model reduction of the discrete time survival analysis showed no significant effect of treatment on the mild cases (OR 1.2; CI 0.65-2.16), but the treated severe UCD lesions showed significantly more improvement compared with the untreated cases (OR 3.4; CI 1.64-7.49; Table 4). The model showed variation in improvement between herds; cows in herds 3 and 4 showed significantly less improvement than cows in herd 1 . Cows with parity $\geq 5$ showed significantly less improvement than cows with parity 1. Finally, less improvement was observed when cows had a dry period observation relative to lactating observations.

\section{DISCUSSION}

To our knowledge this is the first randomized clinical trial to access the clinical effectiveness of 2 topical treatments on mild and severe UCD lesions. Neither of the applied topical treatments contained antibiotics. Recent Dutch research could not show any evidence of primary bacterial involvement (van Engelen et al., 2014). Because of this, and combined with the ongoing belief and necessity of the prudent use of antimicrobials, we decided not to apply topical antibiotic treatments. Taking the position of the lesion into account, topical antibiotic treatment would also increase the risk of contamination of the milk with antibiotics. Because of the similarities between human pressure ulcers and UCD lesions, we focused instead on proven treatments in human medicine; a non-sting alcohol-free barrier film for the mild lesions and an enzyme alginogel with antimicrobial properties for the severe UCD lesions.

Results from the treatment of the severe UCD cases were encouraging. Treatment with the enzyme alginogel showed 3.4 times more improvement of the severe UCD lesions compared with the severe UCD cases in the control group. Current wound therapy in human medicine is based on tissue management, inflammation and infection control, moisture balance, and epithelial 
Table 3. Number of animals with first improvement of udder cleft dermatitis (UCD) lesions after treatment in weeks in treatment and control groups $^{1}$

\begin{tabular}{|c|c|c|c|c|c|c|c|c|c|c|c|c|c|}
\hline Lesion & \multicolumn{13}{|c|}{ No. of animals with first improvement per week } \\
\hline \multicolumn{14}{|c|}{ Treated group } \\
\hline Mild $^{4}$ & 2 & 5 & 2 & 2 & 1 & 2 & 4 & 1 & 0 & 0 & 2 & 0 & $21 / 60$ \\
\hline Severe $A^{5}$ & 5 & 3 & 2 & 1 & 2 & 3 & 0 & 1 & 0 & 1 & 0 & 0 & $18 / 23$ \\
\hline Severe $B^{5}$ & 6 & 1 & 2 & 0 & 0 & 0 & 1 & 0 & 0 & 0 & 0 & 0 & $10 / 24$ \\
\hline Severe $A^{6}$ & 2 & 1 & 0 & 2 & 0 & 0 & 0 & 1 & 0 & 1 & 0 & 1 & $8 / 17$ \\
\hline Severe $B^{6}$ & 1 & 3 & 0 & 0 & 0 & 0 & 0 & 0 & 0 & 0 & 0 & 0 & $4 / 20$ \\
\hline
\end{tabular}

advancement (Hämmerle and Strohal, 2015). These 4 principles exhibit the capacity to stimulate the healing process and are combined in the wound healing property of the used enzyme alginogel. Unfortunately, a treatment period of $12 \mathrm{wk}$ appeared too short to result in the complete healing of the severe lesions, only less than $10 \%$ healed in the follow-up period. Descriptive

Table 4. Results of the discrete time survival analysis model on the time to first improvement in 214 cases of udder cleft dermatitis on 8 dairy herds in the Netherlands

\begin{tabular}{lcc}
\hline Variable & $\begin{array}{c}\text { Odds } \\
\text { ratio }\end{array}$ & $\begin{array}{c}95 \% \\
\text { CI }\end{array}$ \\
\hline Treatment effect & & \\
Treatment mild (vs. untreated mild) & 1.2 & $0.65-2.16$ \\
Treatment severe (vs. untreated severe) & 3.4 & $1.64-7.49$ \\
Status at T0 $^{1}$ (severe vs. mild) & 0.8 & $0.35-1.68$ \\
Other covariate & & \\
Herd & Referent & \\
1 & 1.5 & $0.43-4.50$ \\
2 & 0.2 & $0.06-0.48$ \\
3 & 0.2 & $0.03-0.45$ \\
4 & 0.4 & $0.19-1.01$ \\
5 & 0.9 & $0.43-2.06$ \\
6 & 1.2 & $0.54-2.63$ \\
7 & 0.9 & $0.43-1.77$ \\
8 & & \\
Parity & Referent & \\
1 & 1.4 & $0.70-2.99$ \\
2 & 0.7 & $0.32-1.50$ \\
3 & 0.6 & $0.27-1.41$ \\
4 & 0.3 & $0.11-0.61$ \\
$\geq 5$ & 0.2 & $0.06-0.40$ \\
\hline Dry yes $/$ no $^{2}$ & & \\
\hline
\end{tabular}

${ }^{1} \mathrm{~T} 0=$ recruitment visit.

${ }^{2}$ Dry yes $/$ no $=$ a dry period observation during the trial, analyzed as a time-varying covariate. statistics showed a significantly better first improvement of the smaller class A UCD lesions compared with the class B lesions, uncorrected for potential confounders. Nevertheless, it showed that the size of the lesions has an effect on the likelihood of improvement of the lesion. This is in accordance with human studies which have shown that a greater size and depth of the wound significantly reduces the chances of healing ( $\mathrm{Vu}$ et al., 2007). These findings emphasize the need for early detection followed by prompt treatment. Farmers with herds with a high prevalence of UCD should therefore check their animals every $2 \mathrm{wk}$ for signs of UCD and start with effective treatment before the lesions become larger and thus lengthen the time needed to complete healing. In our study animals with healed lesions were not inspected anymore and therefore we do not have any information about the potential change of relapses of the lesions.

The clinical trial showed no significant effect of treatment on the improvement of mild UCD cases compared with mild UCD cases in the control group. According to the definition, first improvement in mild UCD cases means healing, which was for both the treatment and the control groups $35 \%$. A possible reason for this clinical outcome might be the application method of the film. Farmers treated the affected animals by wiping a small $1 \times 1 \mathrm{~cm}$ tissue saturated with the nonalcoholic film over the lesion. The film was expected to protect the irritated skin against moisture and dirt and was expected to improve the wound healing process. After the study, some farmers complained about the size of the tissue. This size, combined with the hard-to-reach position of the lesion, a large farmer hand, and hectic 
and busy times during milking, may have resulted in an inadequate application of the barrier film. The damaged skin may therefore have been insufficiently protected, leading to less effective healing than expected. Easier ways to apply the film such as bigger tissues or a spray would have been a better way to test the effectiveness of the barrier film, but these were not commercially available.

Results of discrete time survival analysis showed less improvement when cows had a dry period observation relative to lactating observations. At the start of the study, we decided farmers should apply all treatments in the milking parlor. Asking farmers to treat the dry cows as well on a daily or every other day basis in the dry cow pen would have involved a lot of extra work for them, which might have influenced their compliance with the study. As soon as cows were dried off, treatment ceased and was started again directly after parturition. The effect of the dry period was taken into account in the statistical modeling process, but these effects cannot be separated from a nontreatment effect.

At the cow level, we showed that cows with parity 5 or higher showed significantly less improvement than younger animals. Several prevalence and incidence studies (Persson Waller et al., 2014; Bouma et al., 2016; Ekman et al., 2018) showed higher parity as a risk factor for having and developing UCD. Our study suggests that high parity also negatively affects the healing of UCD lesions. It could be that older cows show less or more slowly healing UCD, just as it is the case in other diseases such as subclinical and clinical mastitis (Deluijker et al., 2005; Barkema et al., 2006), probably caused by a less effective immune system of higher parity cows. The udder conformation of older cows could also account for the lower healing rates. The fore udder attachment of cows becomes looser with increasing age, which could lead to moving of the udder skin across the UCD lesion, so called friction. It has been shown that friction impedes the process of wound healing (Bernatchez et al., 2015).

\section{CONCLUSIONS}

Udder cleft dermatitis is a frequently observed lesion of udder skin that affects animal welfare. If successful treatment can improve the healing of UCD lesions, chronicity of the disease could be prevented and the risk of serious health-threatening complications will diminish. In this study, the treatment of mild UCD lesions with a non-sting barrier film did not improve the healing process: first improvements for the treatment and control groups were the same. The treatment of the severe UCD lesions by application of an enzyme alginogel showed a significant improvement in the healing process. Treated animals showed 3.4 times more first improvement of the lesion than the control group. Further research on an effective treatment for mild UCD lesions is still imperative because a fast and successful recovery of mild UCD lesions might prevent transformation of mild UCD lesions into severe UCD lesions. To optimize the effectiveness of treatment of severe UCD lesions, farmers should be advised to check their cows on a regular basis, so lesions can be detected in an early stage and can be followed by prompt treatment. Further studies are needed on the etiology and physiopathology of UCD to recommend preventive measures and to use more targeted treatments, because treatment is only a symptomatic solution for this highly prevalent disease in dairy herds.

\section{ACKNOWLEDGMENTS}

The authors thank the 8 farmers who participated in this study and 3M (Maplewood, MN) and Flen Health (London, UK) for providing their topical treatments. This study was financially supported by the Dutch Dairy Board (Zoetermeer, the Netherlands).

\section{REFERENCES}

Barkema, H. W., Y. H. Schukken, and R. N. Zadoks. 2006. Invited review: The role of cow, pathogen, and treatment regimen in the therapeutic success of bovine Staphylococcus aureus mastitis. J. Dairy Sci. 89:1877-1895. https://doi.org/10.3168/jds.S0022 -0302(06)72256-1.

Beattie, G., and D. J. Taylor. 2000. An investigation into intertrigo (necrotic dermatitis or 'foul udder') in dairy cows. Cattle Pract. 8:377-380.

Beele, H., C. Durante, J. C. Kerihuel, J. Rice, A. Rondas, J. Stryja, and R. White. 2012. Expert consensus on a new enzyme alginogel. Wounds UK, Vol. 8, No. 1.

Bernatchez, S. F., G. E. Mengistu, B. P. Ekholm, S. Sanghi and S. D. Theiss. 2015. Reducing friction on skin at risk: The use of $3 \mathrm{M}^{\mathrm{TM}}$ Cavilon $^{\mathrm{TM}}$ no sting barrier film. Adv. Wound Care (New Rochelle) 4:705-710. https://doi.org/10.1089/wound.2015.0628.

Black, J., M. Baharestani, J. Cuddigan, B. Dorner, L. Edsberg, D. Langemo, M. E. Posthauer, C. Ratliff, and G. Taler. 2007. National Pressure Ulcer Advisory Panel's updated pressure ulcer staging system. Adv. Skin Wound Care 20:269-274. https://doi.org/10 .1097/01.ASW.0000269314.23015.e9.

Bouma, A., M. Nielen, E. van Soest, S. Sietsma, J. van den Broek, T. Dijkstra, and T. van Werven. 2016. Longitudinal study of udder cleft dermatitis in 5 Dutch dairy cattle herds. J. Dairy Sci. 99:4487-4495. https://doi.org/10.3168/jds.2015-9774.

Burnham, K. P., and D. R. Anderson. 2002. Model Selection and Multimodel Inference: A Practical Information-Theoretic Approach. 2nd ed. Springer-Verlag, New York, NY.

Cameron, J., D. Hoffman, J. Wilson, and G. Cherry. 2005. Comparison of two peri-wound skin protectants in venous leg ulcers: An randomized controlled trial. J. Wound Care 14:233-236. https://doi .org/10.12968/jowc.2005.14.5.26779.

Chan, A., and H. Y. Siu. 2016. The use of a no-sting barrier film treatment protocol compared to routine clinical care for the treatment of stage 1 and 2 pressure injuries in long-term care. J. Am. Coll. Clin. Wound Spec. 7:30-34. https://doi.org/10.1016/ j.jccw.2016.11.001. 
Deluijker, H. A., S. N. van Oye, and J. F. Boucher. 2005. Factors affecting cure and somatic cell count after pirlimycin treatment of subclinical mastitis in lactating cows. J. Dairy Sci. 88:604-614. https://doi.org/10.3168/jds.S0022-0302(05)72724-7.

Dohoo, I., W. Martin, and H. Stryhn. 2009. Discrete time survival analysis. Chapter 19.13 in Veterinary Epidemiologic Research. 2nd ed. VER Inc., Charlottetown, PEI, Canada.

Ekman, L., A. K. Nyman, H. Landin, U. Magnusson, and K. P. Waller. 2018. Mild and severe udder cleft dermatitis-Prevalence and risk factors in Swedish dairy herds. J. Dairy Sci. 101:556-571. https:// doi.org/10.3168/jds.2017-13133.

Eming, S. A., T. Krieg, and J. M. Davidson. 2007. Inflammation in wound repair: Molecular and cellular mechanisms. J. Invest. Dermatol. 127:514-525. 10.1038/sj.jid.5700701.

Evans, N. J., D. Timofte, S. D. Carter, J. M. Brown, R. Scholey, D. H. Read, and R. W. Blowey. 2010. Association of treponemes with bovine ulcerative mammary dermatitis. Vet. Rec. 166:532-533. https://doi.org/0.1136/vr.b4822.

Hämmerle, G., and R. Strohal. 2015. Der Einsatz von Enzym Alginogelen in der Therapie schwer heilender chronischer Wunden. J Wundmanagement 2:54-59.

Holroyd, S., and K. Graham. 2014. Prevention and management of incontinence-associated dermatitis using a barrier cream. Br. J. Commun. Nurs. 19(Sup12)S32-S38. https://doi.org/10.12968/ bjen.2014.19.Sup12.S32.

Millar, M., A. Foster, J. Bradshaw, A. Turner, R. Blowey, N. Evans, and G. Hateley. 2017. Embolic pneumonia in adult dairy cattle associated with udder cleft dermatitis. Vet. Rec. 180:205-206. https://doi.org/10.1136/vr.j954.

Olde Riekerink, R. G., K. Van Amersfort, O. C. Sampimon, G. A. Hooijer, and T. J. Lam. 2014. Prevalence, risk factors, and a field scoring system for udder cleft dermatitis in Dutch dairy herds. J. Dairy Sci. 97:5007-5011. https://doi.org/10.3168/jds.2013-7651.

Persson Waller, K., M. Bengtsson, and A. K. Nyman. 2014. Prevalence and risk factors for udder cleft dermatitis in dairy cattle. J. Dairy Sci. 97:310-318. https://doi.org/10.3168/jds.2013-7186.

Silveira Chalita, L. V. A., E. A. Colosimo, and J. Raimundo de Souzza Passos. 2006. Modelling grouped survival data with time-dependent covariates. Commun. Stat. Simul. Comput. 35:975-981.

Stamm, L. V., R. L. Walker, and D. H. Read. 2009. Genetic diversity of bovine ulcerative mammary dermatitis-associated Treponema. Vet. Microbiol. 136:192-196. https://doi.org/10.1016/j.vetmic 2008.10.022

van Engelen, E., N. Meertens, T. Dijkstra, M. Gonggrijp, L. Roos, and A. Velthuis. 2014. Udder Cleft Dermatitis; Onderzoek aanwezige kiemen (Udder Cleft Dermatitis; Study into presence micro-organisms). Eindrapport 1080185 [In Dutch].

Vu, T., A. Harris, G. Duncan, and G. Sussman. 2007. Cost-effectiveness of multidisciplinary wound care in nursing homes: A pseudorandomized pragmatic cluster trial. Fam. Pract. 24:372-379.

Warnick, L. D., D. Nydam, A. Maciel, C. L. Guard, and S. E. Wade. 2002. Udder cleft dermatitis and sarcoptic mange in a dairy herd. J. Am. Vet. Med. Assoc. 221:273-276.

Wilmink, J. M. 2017. Differences in wound healing between horses and ponies. Pages 14-29 in Equine Wound Management, 3rd ed. C. Theoret and J. Schumacher, ed. Wiley Blackwell, Ames, IA.

Winter, G. D. 1962. Formation of the scab and the rate of epithelization of superficial wounds in the skin of the young domestic pig. Nature 193:293-294.

\section{APPENDIX}

\section{Photo I}

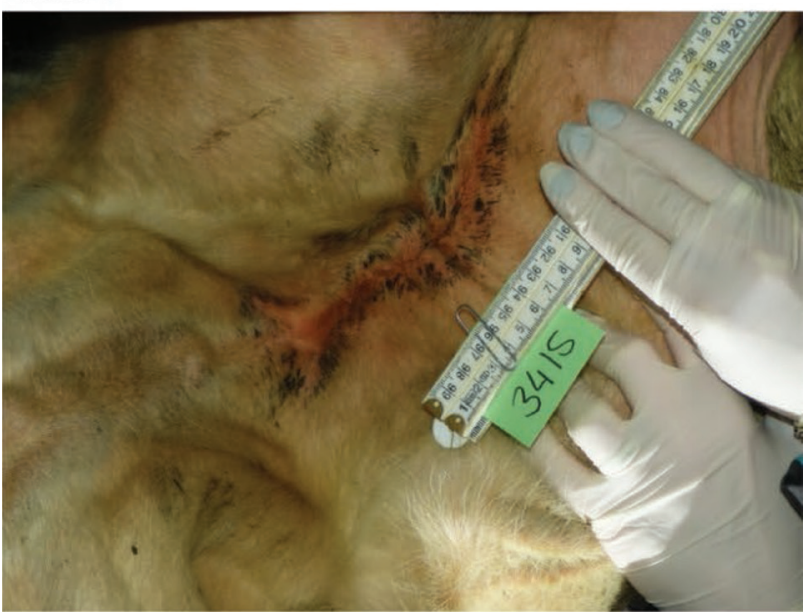

Photo II

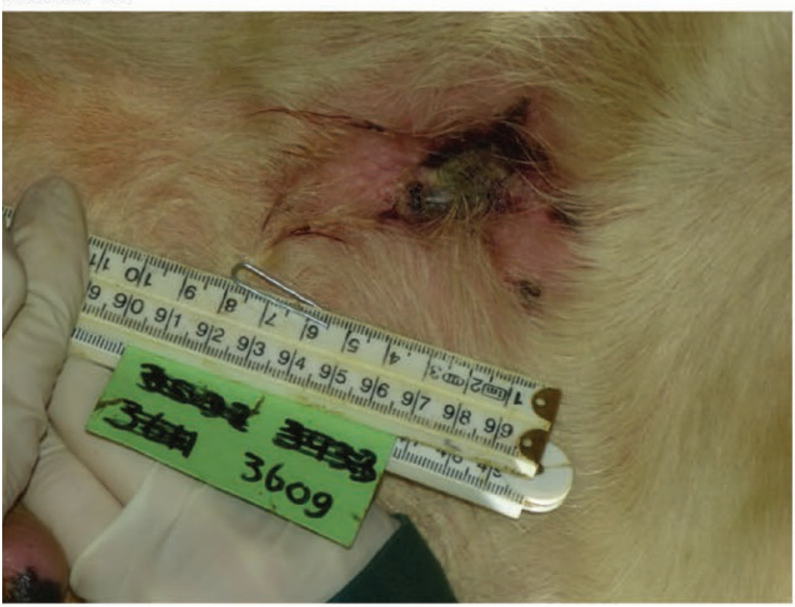

\section{Photo III}

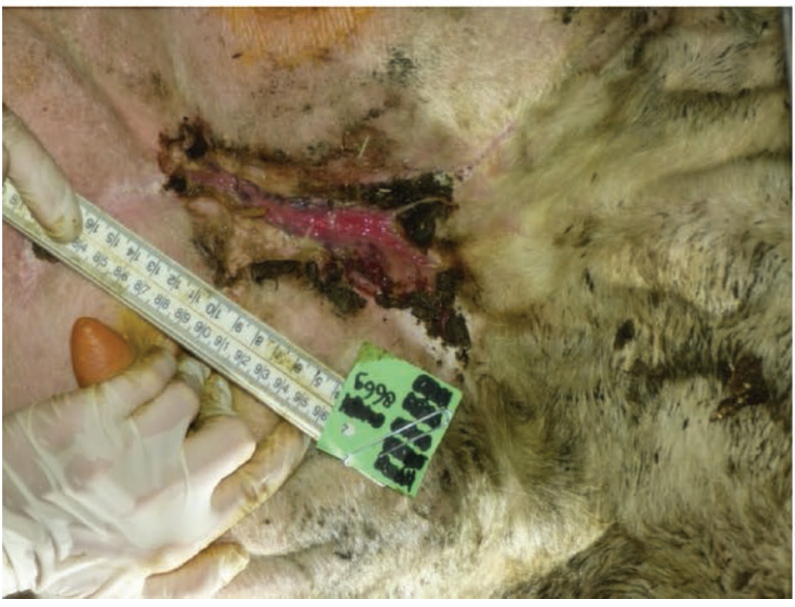

Figure A1. Photographs of the different categories of udder cleft dermatitis (UCD) lesions. Photo I: mild UCD lesion (no wound, intact skin). Photo II: severe class A UCD lesion (lesion length $<5 \mathrm{~cm}$ ). Photo III: severe class B UCD lesion (lesion length $\geq 5 \mathrm{~cm}$ ). Color version available online. 